\title{
Recognition of Leaf Image Set Based on Manifold-Manifold Distance
}

\author{
Ji-Xiang Du ${ }^{1 *}$, Mei-Wen Shao ${ }^{1}$, Chuan-Min Zhai ${ }^{1}$, Jing Wang ${ }^{1}$, Yuanyan Tang ${ }^{2}$, Chun Lung Philip Chen ${ }^{2}$ \\ 1) Department of Computer Science and Technology, Huaqiao University, Xiamen, China \\ 2) Faculty of Science and Technology, Universityof Macau, Macau, China \\ $\{$ jxdu77,wsxxdsmw\}@gmail.com
}

\begin{abstract}
Recognizing plant leaves has been a difficult and important work. In this paper, we formulate the problems by classifying leaf image sets rather than single-shot image, each of set contains leaf images pertaining to the same class. We extract leaf image feature and compute the distance between two manifolds modeled by leaf images. Specifically, we apply a clustering procedure in order to express a manifold by a collection of local linear models. Then the distance is measured between local models which come from different manifolds that constructed above. Finally, the problem is transformed to integrate the distance between pairs of subspace. Experiment based on the leaves (ICL) from intelligent computing laboratory of Chinese academy of sciences, which shows that the method has a great performance.
\end{abstract}

Keywords: Plant leaves classification; Leaf image set; Manifold-Manifold distance; PHOG descriptor

\section{Introduction}

When we wander around the fields, we can find lots of plant. However, we rarely know their names. There are about 270,000 plants that have been named on Earth, and many are still unknown yet. Human identify the large number of existing plant species are dreary and time consuming, particularly for those non-expert. On the other hand, plant recognition or classification has a broad application prospective in the agriculture and medicine, and is especially significant to the biology diversity research. But in recent years people have been seriously destroying the natural environments, so that many plants constantly die and even die out every year. The main step of protecting plants is to automatically recognize them and understand what they are and where they come from. To handle such huge information, develop a quick and efficient classification method has become a significant research.

There are several ways to recognize a plant, like fruit, root, flower, leaf etc. And they are three dimensional objects and have complex with the exception of leaf. Leaf classification and recognition is a significant component of automated plant recognition system, because leaf features often contain important information that can help in plant species recognition and we can obtain a great number of leaves easily. A leaf can be characterized by its color, texture, vein structure, and shape. The type of the vein is an important morphological characteristic of the leaf.

In traditional visual recognition task, leaves of interest are trained and recognized from only a few samples. Rashad et al. [1] have used a combined classifier learning vector quantization along with the radial basis function, a small part of leaf can be classified by the proposed system. Hossain et al. [4] proposed a method which works for the plants with broad flat leaves. In this method, the user selects the base point of the leaf and a few reference points on the leaf blades. On the basis of these points, the leaf shape is extracted from the background and a binary image is produced. Zheng et al [5] extracted leaf vein based on gray scale morphology. The main idea is to look upon the leaf vein as the noise on the leaf surface and adopt the method of noise detection to extract the leaf vein. Lee et al. [10] used the main vein 
and the frequency domain data by using Fast Fourier Transform methods in conjunction with distance measurement between the contour and centroid on detected leaf images. Hu et al [19] propose a novel contour-based shape descriptor, called the multiscale distance matrix, to capture the shape geometry while being invariant to translation, rotation, scaling, and bilateral symmetry. Du et al [13] used a new method of describing the characteristics of plant leaves based on the outline fractal dimension and venation fractal dimension. MG Larese [28] et al proposed a procedure for segmenting and classifying scanned legume leaves based only on the analysis of their veins (leaf shape, size, texture and color are discarded). The segmentation is performed using the unconstrained hit-or-miss transform and adaptive thresholding. Several morphological features are computed on the segmented venation.

After extracting leaf image feature, the classification and recognition task often use traditional classifier that based on single-shot image, such as k-nearest neighbor, learning vector quantization, probabilistic neural network [20-26], support vector machine, genetic algorithm etc. Although the traditional classifier have achieved a certain level of success under restricted conditions in leaf automatic classification and recognition, more robust object recognition can be expected by using sets as input rather than single image, because an image set offers more information compared with a single image. We can extract many leaves from a photo which is taken from a tree or other plants [3]. The quantity of leaf images for both training and testing can be very large. These leaves appearance changes dramatically under variations in shape, size, texture, etc. Therefore we introduce a novel approach for leaf recognition using multiple leaf image patterns obtained in various views.

For image set classification, existing methods mainly concentrate on the key issues of how to model the image sets and haw to measure their similarity. From the view of set modeling, relevant approaches to image set classification almost fall into parametric or nonparametric representations. Nonparametric methods use more flexible manner to assume the distributions of the set data. Kim et al [6] represent the image set as a single linear subspace. Wang et al [12] employ more sophisticated manifold to describe an image set. They use the method of principal angles to capture the similarity of two subspaces. Hakan et al [15] model each set as an affine hull or a convex hull and match the closest pair of points from two hulls.

In this paper we propose a method of recognizing leaf images based on image sets using manifold to manifold distance. As illustrated in figure1, each class of leaves is enrolled with a gallery image set, and the unknown species are also represented by different probe image sets. We model the leaves from one species as a manifold and calculate the distances between pairs of manifolds, identification is achieved by seeking the minimum distance. First of all, we extract local liner models via clustering from different manifolds which are expressed by various species of leaves. Then calculate the similarity between pairs of local models by principal angle and compute the manifold to manifold distance by integrating the similarities. 


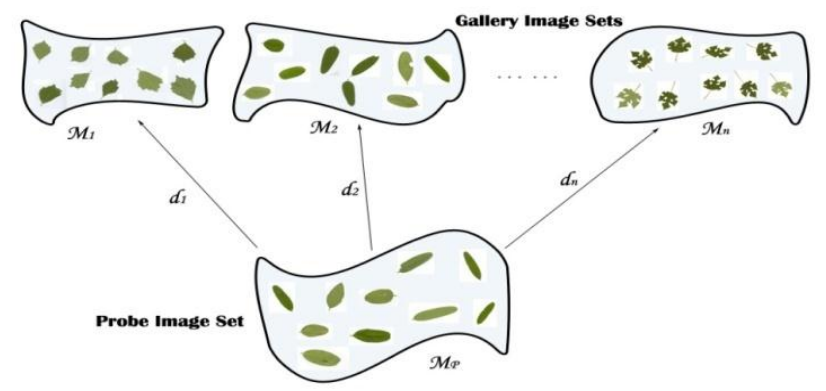

Fig. 1. Leaf recognition based on image sets

\section{Problem Formulation}

In view of the above discussions, we extract shape feature and model the image set as manifold, and then formulate the problem as multi-manifolds learning. Formally, given a leaf database as

$$
G=\left\{X_{1}, X_{2}, \ldots, X_{n}\right\}
$$

where

$$
X_{i}=\left[x_{i, 1}, x_{i, 2}, \ldots, x_{i, n i}\right](i=1, \ldots, n)
$$

express a data matrix of the i-th set, and nidefine as the number of image samples. As mentioned above, we model each image set $X_{i}$ as a nonlinear manifold $M_{i}$ and then extract the local linear models to characterize it, define as:

$$
M_{i}=\left\{L_{i, 1}, L_{i, 2}, \ldots, L_{i, N i}\right\}
$$

Here, $N i$ denotes the number of local models in the i-th set. Typically $N i<<n i$.

Given a probe image set $T=\left\{L_{1}^{\prime}, L_{2}^{\prime}, \ldots, L_{l}^{\prime}\right\}$ containingl local liner models of a species whose identity is one of the $n i$ species in the gallery set. We formulate the problem as follows:

$$
\begin{array}{r}
d\left(M_{i}, T_{j}\right)=\sum_{i=1}^{N i} \sum_{j=1}^{l} f_{i j} d\left(L_{i}, L_{j}^{\prime}\right), \\
\text { s.t. } \sum_{i=1}^{N i} \sum_{j=1}^{l} f_{i j}=1, f_{i j} \geq 0 .
\end{array}
$$

In this general formulation, the manifold to manifold distance comes from a weighted average of pair-wise subspace distance. 


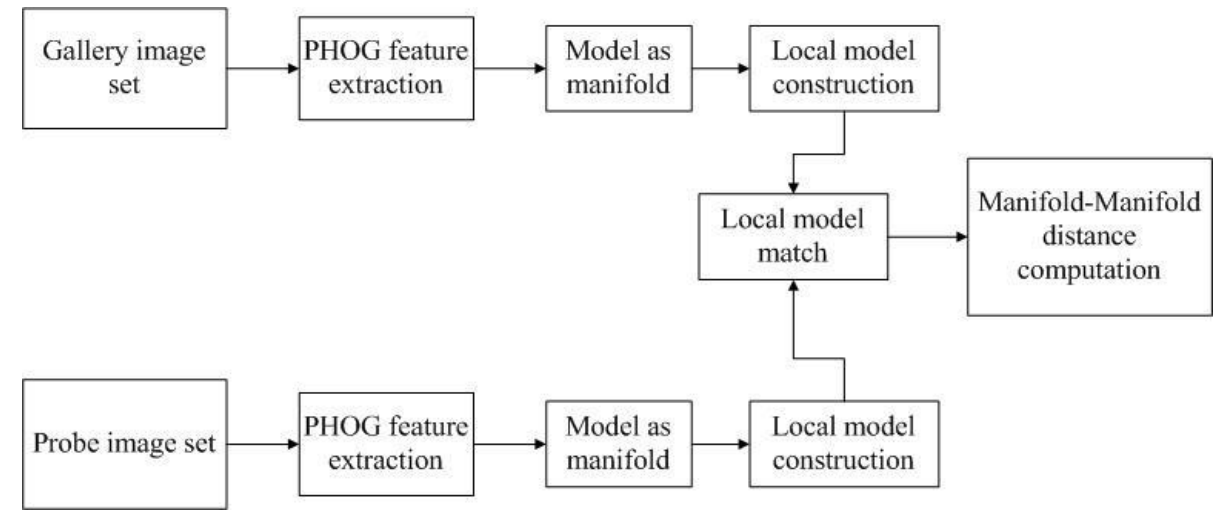

Fig. 2. Framework of our leaf image set recognition system

\section{$3 \quad$ Feature Extraction}

In this paper, we extract leaf shape information by Pyramid of Histograms of Orientation Gradients (PHOG) [7]. PHOG is a spatial pyramid extension of the histogram of gradients descriptors (HOG). The HOG descriptor calculates the occurrences of gradient orientation in localized parts of an image.

In the first, the PHOG descriptor extracts canny edges. Then the leaf image is divided into spatial grids at all pyramid levels. After that, orientation gradients are calculated by the Sobel mask. At last, the gradients of each grid are linked together at each pyramid level. In our experiments, we set the number of pyramids $L=3$ and the bin size $N=8$, the orientation range is $0^{\circ}$ to $360^{\circ}$.

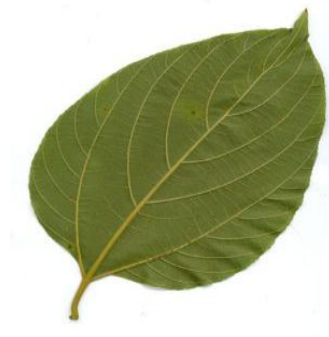

(a)

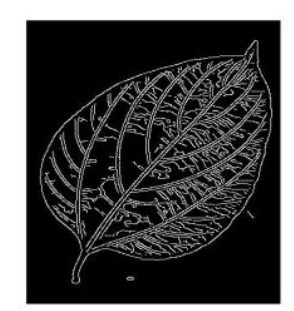

(b)

Fig. 3. PHOG descriptor, (a) Original leaf image, (b) edge orientation figure 

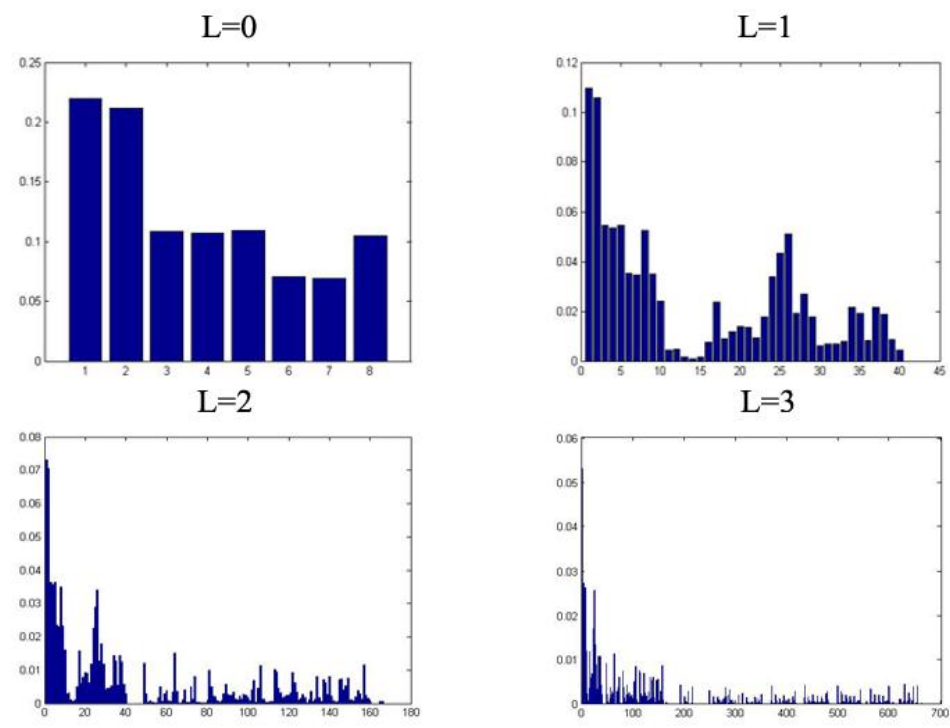

Fig. 4. Histogram representations corresponding to levels $l=0$ to $l=3$

\section{$4 \quad$ Local Liner Model Construction}

To extract local liner models from manifold, the main challenge is how to guarantee the linearity property explicitly [9][27]. In common sense, Euclidean distance is similar to geodesic distance for any two points in a local linear-approximation model. Therefore we employ the deviation between Euclidean distance and geodesic distance to measure the nonlinearity degree in a manifold.

In this thesis, we use the graph-based method of Isomap for approximating the geodesic distances between images in a leaf manifold and employ hierarchical divisive clustering to extract a collection of local models. Firstly, the pair-wise geodesic distance matrix $D_{G}$ and Euclidean distance matrix $D_{E}$ (based on k-NN graph) are computed. Meanwhile, another matrix $H$ is also constructed, each column holding the k-NNs' indices of any points. To measure the nonlinearity degree of one local model, we define a nonlinearity score function as:

$$
S_{i, k}=\frac{1}{n i^{2}} \sum_{m=1}^{n i} \sum_{n=1}^{n i} D_{G}\left(x_{m}, x_{n}\right) / D_{E}\left(x_{m}, x_{n}\right)
$$


This function can effectively guarantee the local linear property and adaptively control the number of local models. The detailed implementation of the algorithm is showed in Algorithm 1.

In this algorithm, the threshold $\delta$ in step 5 controls not only the termination of the algorithm, but also the number of final clusters as well as their nonlinearity degrees. In each iterative step, the cluster in the parent level with the maximum amount of points will be split into two smaller ones with decreased degrees. Why we use this strategy is that in most cases the appropriate number of clusters is much smaller than the number of data samples, and the balanced number of points in each cluster is benefits to our final work, which is integrating the distances between pairs of subspaces.

Then the extracted clusters are represented by linear subspaces to construct the final local models. We employ principal component analysis (PCA) for its simplicity and efficiency. For each local model $L_{i}$, we define its corresponding principal component matrix by $P_{i} \in R^{D \times d_{i}}$ that is computed as the leading eigenvectors of the covariance matrix and forms a set of orthonormal basis of the subspace. Here, the PCA dimension $d_{i}$ is chosen to preserve $90 \%$ variance.

Algorithm 1: Local model construction

1. Given a manifold $M_{i}$, all data points are initialized as a cluster $L_{i, 1}, N i=1$.

2. Compute the number of images in each cluster; $L_{i, k}(k \in\{1,2, \ldots, N i\})$ choose $L_{i, k}$ with the maximum amount of points.

3. Select two furthest seed points according to geodesic distance matrix $D_{G}$ from $L_{i, k}$.Initialize each seed point as a new cluster, $L^{1}$ and $L^{2}$.Update $L^{1}$ and $L^{2}$ by iteratively running step 4 until $L_{i, k}=\varphi$.

4. According to the matrix $H$, gathers the k-NN samples of all the points in $L^{1}$ and $L^{2}$ and merge into them respectively. Update $L_{i, k}$ by deleting the points which are merged into $L^{1}$ and $L^{2}$.

5. The single cluster $L_{i, k}$ splits into two ones and $N i$ plus one; compute the nonlinearity score $S_{i, k}$ according to Eq.(5) for each $L_{i, k}$.If the largest $S_{i . k} \leq \delta$, return the current clustering results; else, go to step 2.

\section{$5 \quad$ Local Model Distance Measure}

\subsection{Principal angles}

Let $P_{1} \in R^{D \times d_{1}}$ and $P_{2} \in R^{D \times d_{2}}$ respectively define the orthonormal basis of two 
subspaces $S_{1}$ and $S_{2}$. The principal angles $0 \leq \theta_{1} \leq \cdots \leq \theta_{m} \leq \pi / 2$ between two subspaces $S_{1}$ and $S_{2}$ are uniquely defined as:

$$
\begin{gathered}
\cos \left(\theta_{k}\right)=\max _{u_{k} \in S_{1}} \max _{v_{k} \in S_{2}} u_{k}^{T} v_{k} \\
\text { s.t. } \quad u_{k}^{T} u_{k}=v_{k}^{T} v_{k}=1, u_{k}^{T} u_{i}=v_{k}^{T} v_{i}=0, i=1,2, \ldots, k-1
\end{gathered}
$$

Where $m=\min \left(\operatorname{dim}\left(S_{1}\right), \operatorname{dim}\left(S_{2}\right)\right)[8] . u_{k}$ and $v_{k}$ are called the k-th pair of canonical vectors. The cosines of the principal angles are called canonical correlations.

A numerically stable algorithm to compute the principal angles was proposed in [16] based on Singular Value Decomposition (SVD):

$$
P_{1}^{T} P_{2}=Q_{12} \Lambda Q_{21}^{T} \quad \text { s.t. } \quad \Lambda=\operatorname{diag}\left(\sigma_{1}, \ldots, \sigma_{r}\right)
$$

where $Q_{12}$ and $Q_{21}$ are orthogonal matrices. The singular values $\sigma_{1}, \ldots, \sigma_{m}$ are the cosines of the principal angles, canonical correlations:

$$
\cos \left(\theta_{k}\right)=\sigma_{k}, k=1,2, \ldots, r \text {. }
$$

The associated canonical vectors are $U=P_{1} Q_{12}=\left[u_{1}, \ldots, u_{d_{1}}\right], \quad V=P_{2} Q_{21}=\left[v_{1}, \ldots, v_{d_{2}}\right]$. In other words, the first principal angle $\theta_{1}$ is the smallest angle between all pairs of unit vectors in the first and the second subspaces. The rest of the principal angles are similarly defined. If the maximum principal angle is small, the subspaces are close to each other. Intuitively, the first pair of canonical vectors corresponds to the most similar modes of variation of two linear subspaces; every next pair to the most similar modes orthogonal to all previous ones.

\subsection{Distances for Subspaces}

Various subspace distances have been defined based on principal angles.

1) Max Correlation

$$
d_{\text {Max }}\left(S_{1}, S_{2}\right)=\left(1-\cos ^{2} \theta_{1}\right)^{1 / 2}=\sin \theta_{1}
$$

In the pioneering study named Mutual Subspace Method (MSM)[14], only use the smallest principal angle $\theta_{1}$ to define a distance.

2) Min Correlation

$$
d_{\text {Min }}\left(S_{1}, S_{2}\right)=\left(1-\cos ^{2} \theta_{m}\right)^{1 / 2}=\sin \theta_{m} .
$$

The min correlation is defined similarly to the max correlation using the largest principal angle $\theta_{r}$. 
3) Projection metric

$$
d_{P}\left(S_{1}, S_{2}\right)=\left(\sum_{i=1}^{m} \sin ^{2} \theta_{i}\right)^{1 / 2}=\left(m-\sum_{i=1}^{m} \cos ^{2} \theta_{i}\right)^{1 / 2} .
$$

The Projection metric is the 2-norm of the sine of all the principal angles [11].

4) Binet-Cauchy metric

$$
d_{B C}\left(S_{1}, S_{2}\right)=\left(1-\prod_{i} \cos ^{2} \theta_{i}\right)^{1 / 2} .
$$

The Binet-Cauchy metric is defined with the product of canonical correlations [2].

The choice of the best distance for a classification task depends on the distribution of data. Since the distances are defined with particular combinations of the principal angles, the best distance depends highly on the probability distribution of the principal angles of the given data. Here we employ the first choice max correlations, it uses the smallest principal angle $\theta_{1}$ only, since it is outperformed on the leaf data and robust when the data are noisy.

\section{Global Integration of Local Distances}

To choose the weights $f_{i j}$ in (4) is our last component of work after computing the distances between subspaces. To match the two sets as the same class, the most effective solution would be to find the common views and measure their similarity rather than matching each pair of local models from two manifolds, those neighboring pairs deserve more emphasis.

Following the notation above, giving two manifolds, we define two indicator functions:

$$
\begin{aligned}
& M_{1}=\left\{L_{i}: i=1, \ldots, m\right\}, M_{2}=\left\{L_{j}^{\prime}: j=1, \ldots, n\right\} \\
& N(i)=\arg \min _{j} d\left(L_{i}, L_{j}^{\prime}\right), j=1, \ldots, n, \\
& N^{\prime}(j)=\arg \min _{i} d\left(L_{i}, L_{j}^{\prime}\right), i=1, \ldots, m .
\end{aligned}
$$

Here, $N(i)$ is an index that defined for local model $L_{i}$ in $M_{1}$, indicating the nearest neighbor $L_{j}^{\prime}$ in $M_{2}$. Similarly, $N^{\prime}(j)$ defined for $M_{2}$ indicates the nearest neighbor index of the local model $L_{j}$ in $M_{1}$. 


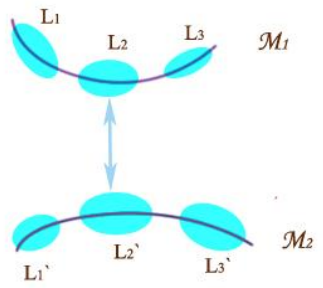

(a)Min-NN

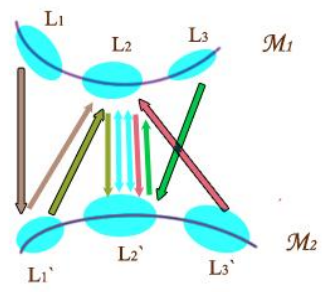

(b) mean-NN's $N N$

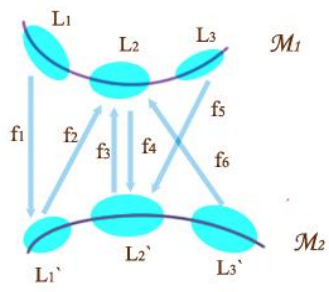

(c) Mean-NN'SIZE

Fig.5. Different global integration options. Both manifolds have three local models and the

Arrows going from each local model to its nearest local model.

Based on finding the common views and measure their similarity, several methods for global integration of local distances have been defined in the literature. For example, the most simple but intuitive method is to measure the similarity between their best suited local models, it just computes the minimum distance among subspace pairs, it is showed in Fig.5 (a) and called Min-NN:

$$
\begin{aligned}
& d\left(M_{1}, M_{2}\right)=\min _{L_{i} \in M_{1} L_{j} \in M_{2}} d\left(L_{i}, L_{j}^{\prime}\right) \\
& =\min \left\{\left.d\left(L_{i}, L_{N(i)}^{\prime}\right)\right|_{i=1} ^{m},\left.d\left(L_{N^{\prime}(j)}, L_{j}^{\prime}\right)\right|_{j=1} ^{n}\right\}
\end{aligned}
$$

Another method transfers some weights from the further pairs to closer ones. It can guarantee more stable results by using information from more data. It is showed in Fig.5 (b) and called mean-NN's $N N$ :

$$
d\left(M_{1}, M_{2}\right)=\frac{1}{m+n}\left\{\sum_{i=1}^{m} d\left(L_{N^{\prime}(N(i))}, L_{N(i)}^{\prime}\right)+\sum_{j=1}^{n} d\left(L_{N^{\prime}(j)}, L_{N\left(N^{\prime}(j)\right)}^{\prime}\right)\right\} .
$$

Both above distances impose more emphasis on those closer pairs, and they do not consider the size (number of points) of each local model. The first method only imposes weight 1 on the closest pair of local models, the integration result may be unstable and affected by outliers easily. The second one although enhances algorithm noise immunity, it just performs well on the condition that the local models extract from subspace have balanced size.

Based on discussion above, we propose a new distance measure, it considers not only the closer pairs but also the size of each pair of subspaces. A more general intuition is that the larger the size of the pair is, the larger its weight should be. This motivates we transfer some weights from the smaller pairs to those larger ones, it is showed in Fig.5 (c) and called Mean-NN'SIZE as follow:

$$
d\left(M_{1}, M_{2}\right)=\frac{1}{\sum_{i=1}^{m} n_{i}+\sum_{j=1}^{n} n_{j}^{\prime}} \times\left\{\sum_{i=1}^{m}\left(n_{i}+n_{N(i)}^{\prime}\right) \times d\left(L_{i}, L_{N(i)}^{\prime}\right)+\sum_{j=1}^{n}\left(n_{N^{\prime}(j)}+n_{j}^{\prime}\right) \times d\left(L_{N^{\prime}(j)}, L_{j}^{\prime}\right)\right\} .
$$

Now it is easy to compute the distance formulated in Eq. (4) from two leaf image sets. For example in Fig.1, each class of leaves is enrolled with a gallery image 
set, and the unknown species are also represented by different probe image sets. We model the leaves from one species as a manifold and calculate the distances between pairs of manifolds, identification is achieved by seeking the minimum distance.

\section{$7 \quad$ Experiments and Results}

Our experiment is conducted on the leaves (ICL) from intelligent computing laboratory of Chinese academy of sciences. The ICL leaf dataset containing 6000 plant leaf images from 200 species which can be meet our need for a large number of plant leaf images. We take 85 classes of plant leaf images, each class includes at least 300 leaf samples, of which half of them are selected randomly as training samples and the remaining half are used for testing samples. Some examples are shown in Fig.5.

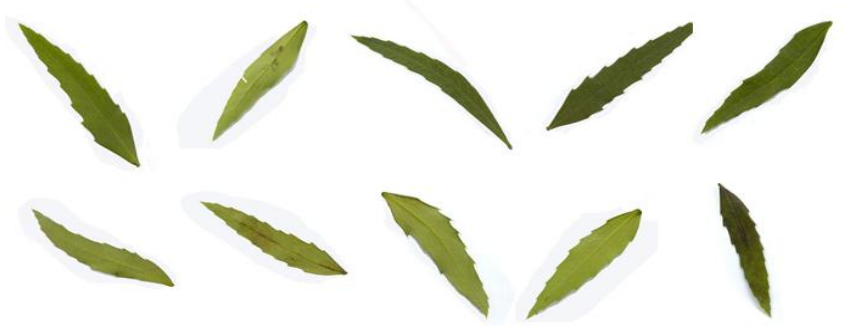

Fig.6. Examples of ICL leaf database, all the leaves come from one class.

The important parameters in our method include the threshold $\delta$ in the hierarchical divisive clustering and the PCA dimension $d_{i}$, which preserves $90 \%$ data energy in our work. Fig.7 shows the average nonlinearity score of all local models in each level. We can see that the average nonlinearity score decreases as the number of local models are increased. Therefore, the chosen of threshold $\delta$ can be the curve ceases to decrease significantly with added local models. This opinion is proved in fig.8, we observe that with maximum nonlinearity degree decreased, the recognition rate increase obviously and the performance is stable when maximum nonlinearity degree is less than 1.3 , this will attribute to the local models representation of the image set manifold. 


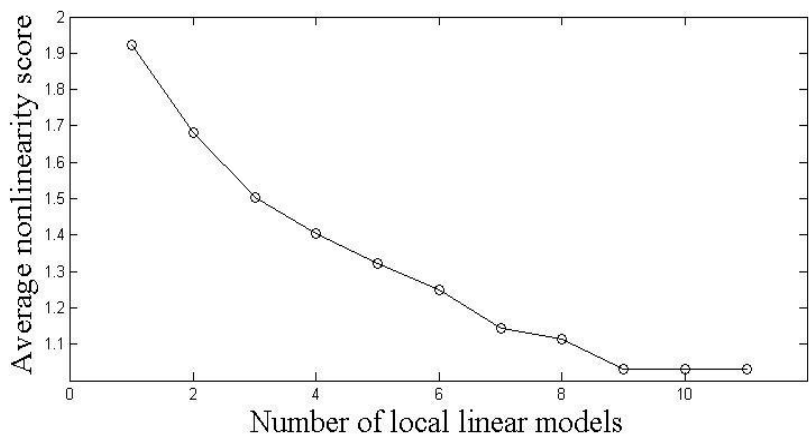

Fig.7. Average nonlinearity score curves, corresponding to an example image set from ICL.

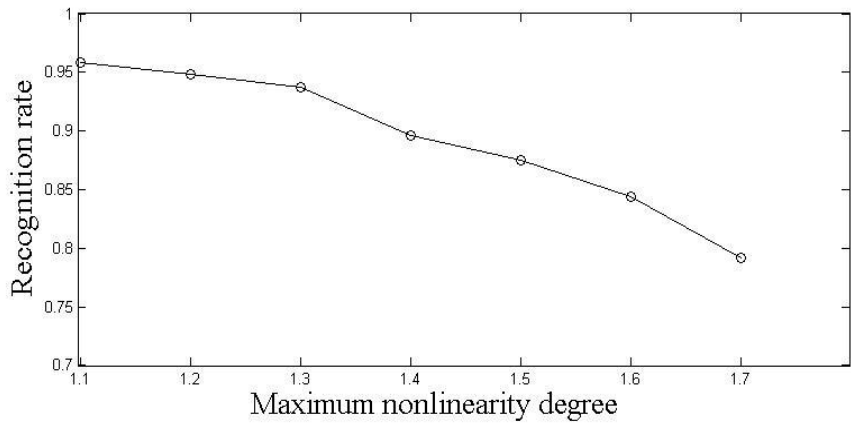

Fig.8. Average recognition rate in different maximum nonlinearity degree

After extracting local liner models from different manifolds, we calculate the similarity between pairs of local models and compute the manifold to manifold distance by integrating the similarities. In this experiment, the threshold $\delta$ fixed at 1.1 and we compare the performance of five subspaces measurement in three global integrations of local distances.

The result is showed in table 1, we can observe that max correlation yields better performance than other measurements which are defined based on principal angels. One possible reason is that each local model is constructed by PCA and max correlation captures the most complicated difference of principal information. Euclidean distance of mean vector and cosine distance of mean vector are exemplar-based measurement, it just tells the position of the subspace in the feature space and merely reflects the difference in the variation modes.

The performance of different global integration of local distance is also showed in table 1, it can be seen the Mean-NN'SIZE distance have the best performance in all subspace measurements. The Min-NN distance only imposes weight 1 on the closest pair of local models and may lose much global information, so the integration result could be poor and unstable. The Mean-NN'NN distance is more stable by using information from more data but without considering the size of different local models. 
The Mean-NN'SIZE distance can adaptively adjust the weights on different size of NN pairs, therefore, it is more reliable and overcome the defect of the former method.

Table 1.Performance comparisons of different subspaces measurement in three global distances

\begin{tabular}{l|c|c|c}
\hline \multirow{2}{*}{$\begin{array}{l}\text { Different distances for } \\
\text { subspaces }\end{array}$} & \multicolumn{3}{|c}{ Recognition rates of different global integration of local distances } \\
\cline { 2 - 4 } & Min-NN & mean-NN's NN & Mean-NN'SIZE \\
\hline Max Correlation & 86.5 & 91.7 & 95.8 \\
\hline Min Correlation & 7.3 & 7.3 & 15.6 \\
\hline Projection metric & 59.4 & 79.2 & 90.7 \\
\hline $\begin{array}{l}\text { Euclidean distance of } \\
\text { mean vector }\end{array}$ & 73.1 & 76.9 & 81.2 \\
\hline $\begin{array}{l}\text { Cosine distance of } \\
\text { mean vector }\end{array}$ & 76.9 & 78.8 & 79.2 \\
\hline
\end{tabular}

After evaluating the performance of three ingredients in our leaf image set recognition method, we compared the performance of different classification methods, include the sample-based methods and set-based methods.

1. The most classical sample-based method K-Nearest neighbor (K-NN), K equal to 3 in our experiment.

2. Nearest neighbor matching by LDA which is classical discriminate analysis method. In order to avoid singularity problems, we adopted the methods in accordance with [18].

3. Support Vector Machine (SVM), we employ Radial basis function kernel.

4. The typical exemplar-based method $\mathrm{NN}$ matching in LLE+k-means clustering [17], we use the same parameters setting as [17].

5. The typical image set recognition method Mutual Subspace Method (MSM), we apply PCA to get subspace basis of each image set and preserve $95 \%$ data energy, then the max correlation measurement is used to calculate the subspace distance.

6. In our method, we apply Mean-NN'SIZE global integration distance and Max Correlation subspace measurement, the threshold $\delta=1.1$.

The average recognition rates for the compared experiments are demonstrated in Table 2. It can be seen the sample-based methods K-NN and LDA yield relatively poor performance, though SVM classifier has better performance, it still perform worse than image set classification method. This is because the leaf image sets contain large shape variations and the sample-based methods may not work well in a complex environment. In the other three set based method, LLE+K-means exhibits the lowest recognition rates, since it uses exemplars to represent clusters which are exploited from a manifold. Though the exemplar-based method is simple, it just tells the position of the subspace in the feature space and may not characterize the variation of the leaf image set well. 
Both MSM and manifold to manifold distance employ principal angles to calculate the similarity between two subspaces, but the MSM just model the whole image set as a liner subspace. In contrast, the proposed method treats both leaf image sets as nonlinear manifolds, it may be more reasonable and better representation of leaf samples.

Table 2. Performance comparisons of different classifiers

\begin{tabular}{l|c}
\hline & Average identification rate (\%) \\
\hline K-NN & 64.4 \\
\hline LDA & 67.4 \\
\hline SVM & 82.7 \\
\hline LLE+K-means & 78.2 \\
\hline MSM & 84.6 \\
\hline Our method & 95.8 \\
\hline
\end{tabular}

We further evaluated the two common problems in set-based classification. The first problem is the unbalanced set size and lack of data in each set. We simulate the problem by randomly removing certain number of images and keeping $90 \%$ to $30 \%$ samples in each set.

The identification performance for effect of reduced leaf image set is demonstrated in Fig. 9. The dotted line shows the effect of unbalanced set size and the broken line expresses the influence when the gallery and probe image set both have the reduced sample size. In general, with the set size decreasing, the number of resulted local models also drops accordingly. The results show that the identification rate of reduced gallery and test set fall faster than lessened test set. The performance of our algorithm is increasingly poor when we keep less than $70 \%$ samples in each image set, because it gives the global coordinates of the sparse data.

Another common challenge is that the image sets contain noisy data in real world application. Therefore, we conducted two experiments in which the probe sets and both gallery and probe sets are systematically corrupted by adding different number of images from each of the other classes.

From the result in Fig.10, it is observed that our method shows relatively slight performance drops and is robust against the noisy data. This can be mainly attributed to the subspace based modeling and matching. We have three options in the global integration part. The first one is Min-NN, it only computes the closest pair of local models, so the result would be easily affected by outliers. Different from the first option, the remaining two options are more stable by using information from more data. In each image set, the number of noisy data is relatively small and they are distributed far away from the center of manifold, so they would have small weight in (4) when we employ the Mean-NN'SIZE option. 


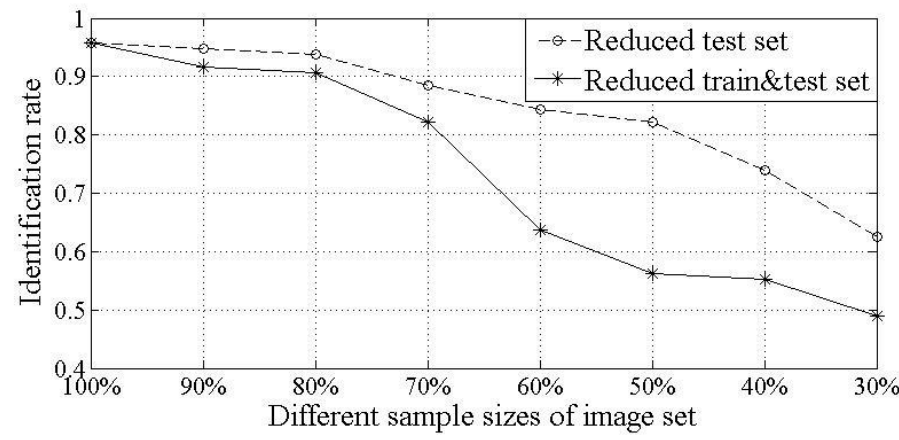

Fig.9. Effect of reduced leaf image set

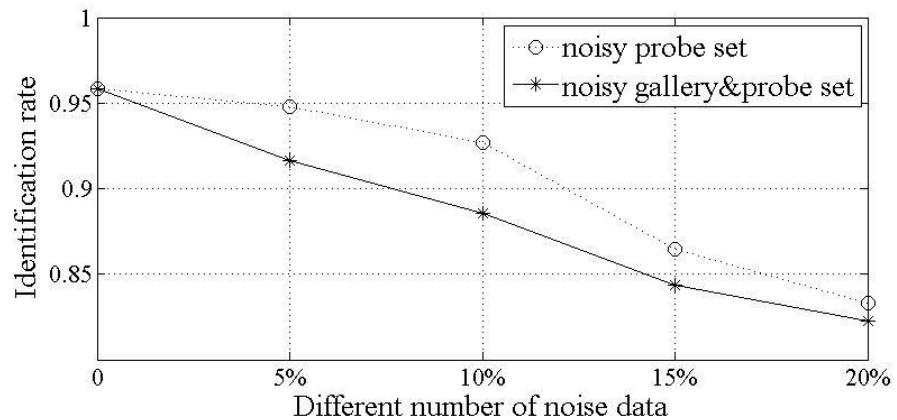

Fig.10. Effect of noisy data on different set

\section{$7 \quad$ Conclusion}

In this work we developed method for leaf recognition from sets of images (rather than from individual images). We extract leaf shape information by PHOG descriptor, and then characterize each image set from the gallery and the probe set as a manifold in feature space. Recognition is performed by finding the minimum distance between manifolds. Though the PHOG is a simple shape descriptor, it has a good result with the set-based classifier. The experiments show that the set-based methods have better performance than traditional sample-based methods in plant leaf recognition task, but each image set requires relatively dense data. Our future research works will include how to classify the leaves with a limited number of images in each image set and improve robustness to noisy data.

Acknowledgments:This work was supported by the Grant of the National Science Foundation of China (No. 61175121), the Program for New Century Excellent Talents in University (No.NCET-10-0117), the Grant of the National Science Foundation of Fujian Province (No.2013J06014), the Program for Excellent Youth Talents in 
University of Fujian Province (No.JA10006), the Promotion Program for Young and Middle-aged Teacher in Science and Technology Research of Huaqiao University (No.ZQN-YX108), the Project of Science and Technology Innovation Platform of Fujian province (NO.2012H2002).

\section{References}

[1] M.Z. Rashad, B.S el-Desouky., and M.S. Khawasik. Plants Images Classification Based on Textural Features using Combined Classifier. International Journal of Computer Science \& Information Technology (IJCSIT), 3(4), (2011), 93-100.

[2] Wolf, Lior, and Amnon Shashua. Learning over sets using kernel principal angles. The Journal of Machine Learning Research , 4, (2003), 913-931.

[3] X.F. Wang, D.S. Huang, and H. Xu. An efficient local Chan-Vese model for image segmentation. Pattern Recognition, 43(3), (2010), 603-618.

[4] Hossain, Javed, and M. Ashraful Amin. Leaf shape identification based plant biometrics. Computer and Information Technology (ICCIT), 2010 13th International Conference on, (2010), 458-463.

[5] X. Zheng, and X. Wang. Leaf vein extraction based on gray-scale morphology. International Journal of Image, Graphics and Signal Processing (IJIGSP), 2, (2010), 25-31.

[6] Kim, Tae-Kyun, Josef Kittler, and Roberto Cipolla. Discriminative learning and recognition of image set classes using canonical correlations. Pattern Analysis and Machine Intelligence, IEEE Transactions on, 29(6), (2007), 1005-1018.

[7] Bosch, Anna, Andrew Zisserman, and Xavier Munoz. Representing shape with a spatial pyramid kernel. Proceedings of the 6th ACM international conference on Image and video retrieval. ACM, (2007), 401-408.

[8] Hotelling, Harold. Relations between two sets of variates. Biometrika, (1936), 321-377.

[9] X.-F. Wang, and D.S. Huang. A novel density-based clustering framework by using level set method. IEEE Trans. Knowledge and Data Engineering, 21(11), (2009), 1515-1531.

[10] Lee, Kue-Bum, Kwang-Woo Chung, and Kwang-Seok Hong. An implementation of Leaf Recognition System. (2013), 152-155.

[11] Edelman, Alan, Tomás A. Arias, and Steven T. Smith. The geometry of algorithms with orthogonality constraints. SIAM journal on Matrix Analysis and Applications, 20(2), (1998), 303-353. 
[12] R. Wang, S. Shan, X. Chen, et al. Manifold-manifold distance and its application to face recognition with image sets. Image Processing, IEEE Transactions on, 21(10), (2012), 4466-4479.

[13] Ji-xiang Du, Chuan-Min Zhai, and Qing-Ping Wang. Recognition of plant leaf image based on fractal dimension features. Neurocomputing ,116, (2013),150-156.

[14] Yamaguchi, Osamu, Kazuhiro Fukui, and Ken-ichi Maeda. Face recognition using temporal image sequence. Automatic Face and Gesture Recognition, 1998. Proceedings. Third IEEE International Conference on. IEEE, (1998), 318-323.

[15] Cevikalp, Hakan, and Bill Triggs. Face recognition based on image sets. Computer Vision and Pattern Recognition (CVPR), 2010 IEEE Conference on. IEEE, (2010), 2567-2573.

[16] Björck, Ȧke, and Gene H. Golub. Numerical methods for computing angles between linear subspaces. Mathematics of computation, 27(123), (1973), 579-594.

[17] Hadid, Abdenour, and M. Pietikainen. From still image to video-based face recognition: an experimental analysis. Automatic Face and Gesture Recognition, 2004. Proceedings. Sixth IEEE International Conference on. IEEE, (2004), 813-818.

[18] Yan, Shuicheng, et al. Graph embedding and extensions: a general framework for dimensionality reduction. Pattern Analysis and Machine Intelligence, IEEE Transactions on, 29(1), (2007), 40-51.

[19] R. Hu, W. Jia, H. Ling, et al. Multiscale distance matrix for fast plant leaf recognition. Image Processing, IEEE Transactions on, 21(11), (2012), 4667-4672.

[20] D.S. Huang, and J.-X. Du, A constructive hybrid structure optimization methodology for radial basis probabilistic neural networks. IEEE Trans. Neural Networks, 19(12), (2008), 2099-2115.

[21] D.S. Huang. Radial basis probabilistic neural networks: Model and application. International Journal of Pattern Recognition and Artificial Intelligence, 13(7), (1999), 1083-1101.

[22] L. Shang, D.S. Huang, J.-X. Du, and C.-H. Zheng. Palmprint recognition using Fast ICA algorithm and radial basis probabilistic neural network. Neurocomputing, 69(13), (2006), 1782-1786.

[23] Z.-Q. Zhao, D.S. Huang, and B.-Y. Sun. Human face recognition based on multiple features using neural networks committee. Pattern Recognition Letters, 25(12), (2004), 1351-1358.

[24] D.S. Huang, Horace H.S.Ip and Z.-R. Chi. A neural root finder of polynomials based on root moments. Neural Computation, 16(8), (2004), 1721-1762. 
[25] D.S. Huang. A constructive approach for finding arbitrary roots of polynomials by neural networks. IEEE Trans. on Neural Networks, 15(2), (2004), 477-491.

[26] A.H. Kulkarni, H.M. Rai, K.A. Jahagirdar, et al. A Leaf Recognition Technique for Plant Classification Using RBPNN and Zernike Moments. International Journal of Advanced Research in Computer and Communication Engineering, 2(1), (2013), 1-5.

[27] B. Li, and D.S. Huang. Locally linear discriminant embedding: An efficient method for face recognition. Pattern Recognition, 41(12), (2008), 3813-3821.

[28] M.G. Larese, R. Namías, R.M. Craviotto, et al. Automatic classification of legumes using leaf vein image features. Pattern Recognition, 47(1), (2014), 158-168. 